\title{
Electromagnetic Scattering from Slightly Rough Surfaces with Inhomogeneous Dielectric Profiles
}

\author{
Kamal Sarabandi, Senior Member, IEEE and Tsenchieh Chiu
}

\begin{abstract}
Remote sensing of soil moisture using microwave sensors require accurate and realistic scattering models for rough soil surfaces. In the past, much effort has been devoted to the development of scattering models for either perfectly conducting or homogeneous rough surfaces. In practice, however, the permittivity of most soil surfaces is nonuniform, particularly in depth, for which analytical solution does not exist. The variations in the permittivity of a soil medium can easily be related to its soil moisture profile and soil type using the existing empirical models. In this paper, analytical expressions for the bistatic scattering coefficients of soil surfaces with slightly rough interface and stratified permittivity profile are derived. The scattering formulation is based on a new approach where the perturbation expansion of the volumetric polarization current instead of the tangential fields is used to obtain the scattered field. Basically, the top rough layer is replaced with an equivalent polarization current and, using the volumetric integral equation in conjunction with the dyadic Green's function of the remaining stratified half-space medium, the scattering problem is formulated. Closed-form analytical expressions for the induced polarization currents to any desired order are derived, which are then used to evaluate the bistatic scattered fields up to and including the third order. The analytical solutions for the scattered fields are used to derive the complete second-order expressions for the backscattering coefficients as well as the statistics of phase difference between the scattering matrix elements. The theoretical results are shown to agree well with the backscatter measurements of rough surfaces with known dielectric profiles and roughness statistics.
\end{abstract}

Index Terms-Electromagnetic scattering, inhomogeneous media, rough surfaces.

\section{INTRODUCTION}

$\mathbf{S}$ OIL moisture, and its temporal and spatial variations are influential parameters in both climatic and hydrologic models. Soil dielectric constant at microwave frequencies exhibits a strong dependence on the soil's moisture content. At $L$ band, for example, the real part of the dielectric constant ranges from three for dry soil to about 25 for saturated soil. This variation can result in a change on the order of $10 \mathrm{~dB}$ in the magnitude of the radar backscatter coefficient [1]. With the advent of the polarimetric synthetic aperture radar (SAR), radar remote sensing of soil moisture has attained significant prominence in the past decade. SAR systems are capable of producing the backscatter map of the terrain with high resolution from an airborne or space-borne platform. From the electromagnetic point of view, remote sensing of soil moisture,

Manuscript received May 3, 1996; revised January 23, 1997. This work was supported by NASA under Contract NAGW-2151.

The authors are with the Department of Electrical Engineering and Computer Science, Radiation Laboratory, University of Michigan, Ann Arbor, MI 48109 USA.

Publisher Item Identifier S 0018-926X(97)06280-7. in the absence of vegetation cover, can be modeled as an inverse scattering problem, where the dielectric constant and surface roughness statistics are to be determined from a set of backscatter measurements.

The problem of wave scattering from random rough surfaces has been the subject of ongoing research over the past several decades because of its applications in many areas of science and engineering. Generally speaking, the available electromagnetic scattering models can be categorized into three major groups: 1) analytical; 2) empirical; and 3) numerical. The analytical scattering solutions for rough surfaces apply when the roughness dimensions of the surface are either much smaller or much larger than the wavelength. For surfaces with small surface root mean square (rms) height and slope, the small perturbation model (SPM) is the most commonly used formalism [2], [3]. In this approach, the surface fields are expanded in terms of a power series in the small roughness parameter and, then, using either the Rayleigh hypothesis or the extended boundary condition [4], the expansion coefficients are obtained recursively. The scattering formulations based on SPM exist for dielectric and perfectly conducting rough surfaces. For these surfaces, only first-order expressions for the copolarized and second-order expressions for the cross-polarized backscattering coefficients are reported [5]. On the other hand, if the irregularities of the surface have relatively small slopes and large radii of curvature, the Kirchhoff approximation (KA) can be used [6]. In this approach, the surface fields at a given point are approximated by those of the local tangent plane. In the past two decades, many attempts have been made to extend the validity region of SPM and KA. Among these, the phase perturbation method (PPM) [7] and the unified perturbation expansion (UPE) [8] for extending the low-frequency techniques and the integral equation method (IEM) [9] for extending the high-frequency techniques can be mentioned. In the PPM, the perturbation solution is obtained by expanding the phase of the field instead of the field itself, whereas in the UPE method, the solution is obtained by expanding the field in terms of a parameter (momentum transfer) that remains small over a region larger than the perturbation parameter used in SPM. Scattering formulations based on PPM and UPM are reported only for one-dimensional rough surfaces. The scattering solution based on the integral equation (IE) method is obtained by inserting the KA into the surface field integral equation. This method is significant in that it reduces to the SPM solution, thereby seemingly bridging the gap between the low- and high-frequency solutions [10]. 
In this paper, no attempt is made to extend the validity region of the existing methods; instead another practical aspect of the scattering problem is investigated. In most practical situations, the soil moisture content as a function of depth is nonuniform in depth. The soil moisture profile is usually a complex function of soil type, temperature profile, surface evaporation, and moisture content [14]. For radar remote sensing of soil moisture, the effect of the inhomogeneity in the complex permittivity of the soil surface on its backscatter must be understood. For this purpose, analytical expressions for the bistatic scattering coefficients of a slightly rough surface with inhomogeneous dielectric profile are derived. Using the classical perturbation expansion of the electric field, a new volumetric integral equation approach is used to obtain the iterative scattering solutions. In what follows, the theoretical formulation for the scattering problem is given and the closed-form complete second-order solution for backscattering coefficients and phase-difference statistics are derived. In Section III, the theoretical solution will be compared with experimental backscatter measurements collected using the University of Michigan's (Ann Arbor) bistatic indoor facilities.

\section{TheORETICAL ANALYSIS}

Consider an inhomogeneous half-space medium with a rough interface as shown in Fig. 1. In the following derivation, it is assumed that the medium is stratified, that is, the relative permittivity is only a function of $z$, and is given by

$$
\epsilon_{r}(x, y, z)=\epsilon_{r}(z) \text {. }
$$

Suppose a plane wave is illuminating the rough interface from the upper medium and, with a very high probability, the surface height variation is small compared with the wavelength of the incident wave. To make the solution tractable, the permittivity of the top layer down to a depth of $d$ is considered to be uniform where $-d<\min \{$ surface profile $\}$. Denote the surface height profile by the function $z=\Delta f(x, y)$ where $f(x, y)$ is a zero-mean stationary random process with a known autocorrelation function and $\Delta \ll \lambda$ is a small constant known as the perturbation parameter. The incident wave can be written as

$$
\mathbf{E}^{i}(\bar{r})=\mathbf{P}_{i} e^{i k_{0} \hat{k}^{i} \cdot \mathbf{r}}
$$

where $\mathbf{P}_{i}$ denotes the polarization of the incident wave, $k_{0}=$ $2 \pi / \lambda$ is the free-space propagation constant, and $\hat{k}^{i}$ is the unit vector along the direction of propagation given by

$$
\hat{k}^{i}=\sin \theta_{i} \cos \phi_{i} \hat{x}+\sin \theta_{i} \sin \phi_{i} \hat{y}-\cos \theta_{i} \hat{z} .
$$

In the absence of the top homogeneous rough layer (with thickness $d$ ), the incident wave would be reflected at the smooth interface between the free-space and the inhomogeneous halfspace soil medium. The reflected wave can be expressed by

$$
\mathbf{E}^{r}(\bar{r})=\mathbf{P}_{r} e^{i k_{0} \hat{k}^{r} \cdot \mathbf{r}}
$$

where $\hat{k}^{r}$ is the direction of propagation of the reflected wave given by

$$
\hat{k}^{r}=\hat{k}^{i}-2\left(\hat{z} \cdot \hat{k}^{i}\right) \hat{z}
$$

and $\mathbf{P}_{r}$ is the polarization vector of the reflected wave, which can be obtained from

$$
\mathbf{P}_{r}=r_{v}\left(\mathbf{P}_{i} \cdot \hat{v}_{i}\right) \hat{v}_{r}+r_{h}\left(\mathbf{P}_{i} \cdot \hat{h}_{i}\right) \hat{h}_{r} .
$$

Here, $r_{v}$ and $r_{h}$ are the Fresnel reflection coefficients and the horizontal and vertical unit vectors are given by

$$
\begin{aligned}
& \hat{h}_{s}=\frac{\hat{k}_{s} \times \hat{z}}{\left|\hat{k}_{s} \times \hat{z}\right|} \\
& \hat{v}_{s}=\hat{h}_{s} \times \hat{k}_{s}
\end{aligned}
$$

where the subscript $s$ can be $i$ or $r$ for the incident and reflected waves. In presence of the homogeneous rough layer, the incident and reflected waves induce a polarization current within the top dielectric layer which is the source of the scattered field. The polarization current in terms of the total field and the permittivity of the layer is

$$
\mathbf{J}(\mathbf{r})=-i k_{0} Y_{0}(\epsilon-1) \mathbf{E}^{t}
$$

where $Y_{0}=1 / Z_{0}$ is the characteristic admittance of the free-space and

$$
\mathbf{E}^{t}=\mathbf{E}^{i}+\mathbf{E}^{r}+\mathbf{E}^{s} .
$$

The scattered field $\mathbf{E}^{s}$ can in turn be expressed in terms of the polarization current and is given by

$$
\mathbf{E}^{s}=i k_{0} Z_{0} \int_{V_{s l a b}} \overline{\overline{\mathbf{G}}}\left(\mathbf{r}, \mathbf{r}^{\prime}\right) \cdot \mathbf{J}\left(\mathbf{r}^{\prime}\right) d v^{\prime}
$$

where $\overline{\overline{\mathbf{G}}}\left(\mathbf{r}, \mathbf{r}^{\prime}\right)$ is the dyadic Green's function of the halfspace inhomogeneous medium (in the absence of the top rough layer). Substituting (3) into (1), the following integral equation for the polarization current can be obtained:

$$
\begin{aligned}
\frac{1}{\epsilon-1} \mathbf{J}(\mathbf{r})= & -i k_{0} Y_{0}\left(\mathbf{E}^{i}+\mathbf{E}^{r}\right)+k_{0}^{2} \iint_{-\infty}^{\infty} \\
& \cdot \int_{0}^{d+\Delta f\left(x^{\prime}, y^{\prime}\right)} \overline{\overline{\mathbf{G}}}\left(\mathbf{r}, \mathbf{r}^{\prime}\right) \cdot \mathbf{J}\left(\mathbf{r}^{\prime}\right) d v^{\prime} .
\end{aligned}
$$

An approximate solution for the integral equation can be obtained using a perturbation technique. By breaking the $z^{\prime}$ integral into two integrals, one with limits from zero to $d$ and the other with limits from $d$ to $d+\Delta f\left(x^{\prime}, y^{\prime}\right)$ and, noting $\Delta f\left(x^{\prime}, y^{\prime}\right)$ is a small quantity, up to the $N$ th order in $\Delta$, (4) can be written as

$$
\begin{aligned}
& \frac{1}{\epsilon-1} \mathbf{J}(\mathbf{r}) \simeq-i k_{0} Y_{0}\left(\mathbf{E}^{i}+\mathbf{E}^{r}\right)+k_{0}^{2} \iint_{-\infty}^{\infty} \int_{0}^{d} \overline{\overline{\mathbf{G}}}\left(\mathbf{r}, \mathbf{r}^{\prime}\right) \\
& \cdot \mathbf{J}\left(\mathbf{r}^{\prime}\right) d x^{\prime} d y^{\prime} d z^{\prime}+k_{0}^{2} \sum_{n=0}^{N-1} \iint_{-\infty}^{\infty} \\
& \cdot \frac{\left[\Delta f\left(x^{\prime}, y^{\prime}\right)\right]^{n+1}}{(n+1) !} \frac{\partial^{n}}{\partial z^{\prime n}} \\
& \cdot\left\{\overline{\overline{\mathbf{G}}}\left(\mathbf{r}, \mathbf{r}_{d}^{\prime}\right) \mathbf{J}\left(\mathbf{r}_{d}^{\prime}\right)\right\} d x^{\prime} d y^{\prime}
\end{aligned}
$$




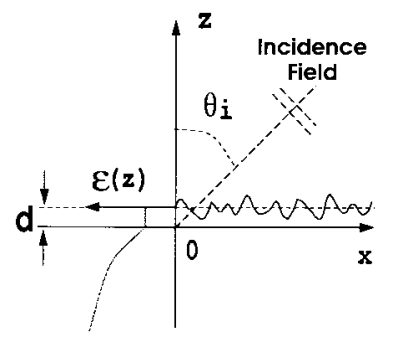

Fig. 1. An inhomogeneous half-space medium with a rough interface. Left side of this figure shows the dielectric profile.

where $\mathbf{r}_{d}^{\prime}=x^{\prime} \hat{x}+y^{\prime} \hat{y}+d \hat{z}$. Taking the two-dimensional Fourier transform of both sides of (5) and noting that the integrals in $x^{\prime}$ and $y^{\prime}$ are of convolution type, it can be shown that

$$
\begin{aligned}
& \frac{1}{\epsilon-1} \tilde{\mathbf{J}}\left(\mathbf{k}_{\perp}, z\right) \\
& =-i 4 \pi^{2} k_{0} Y_{0} \delta\left(\mathbf{k}_{\perp}-\mathbf{k}_{\perp}^{i}\right)\left[\mathbf{P}_{i} e^{-i k_{z}^{i} z}+\mathbf{P}_{r} e^{i k_{z}^{i} z}\right] \\
& \quad+k_{0}^{2} \int_{0}^{d} \tilde{\mathbf{G}}\left(\mathbf{k}_{\perp} ; z, z^{\prime}\right) \cdot \tilde{\mathbf{J}}\left(\mathbf{k}_{\perp}, z^{\prime}\right) d z^{\prime} \\
& \quad+k_{0}^{2} \sum_{n=0}^{N-1} \sum_{m=0}^{n} \frac{\left(\begin{array}{c}
n \\
m
\end{array}\right) \Delta^{n+1}}{(n+1) !} \cdot \frac{\partial^{m}}{\partial z^{\prime m}} \tilde{\mathbf{G}}\left(\mathbf{k}_{\perp} ; z, d\right) \\
& \quad \cdot\left[\frac{\partial^{n-m}}{\partial z^{\prime n-m}} \tilde{\mathbf{J}}\left(\mathbf{k}_{\perp}, d\right) * \bigotimes^{n+1} F\left(\mathbf{k}_{\perp}\right)\right]
\end{aligned}
$$

where $*$ is the convolution operator, $F\left(\mathbf{k}_{\perp}\right)$ is the Fourier transform of $f\left(x_{n}^{\prime}, y^{\prime}\right), \otimes^{n}$ represents $n$-fold self-convolution $(\otimes^{n} F=\overbrace{F * F * \cdots * F}^{n}), k_{z}^{i}=k_{0} \cos \theta_{i}$, and $\tilde{\tilde{\mathbf{G}}}\left(\bar{k}_{\perp} ; z, z^{\prime}\right)$ is the Fourier transform of the Green's function, given by (7), shown at the bottom of the page. In (7), $k_{z}=\sqrt{k^{2}-k_{x}^{2}-k_{y}^{2}}$, $\mathbf{k}_{\perp}=k_{x} \hat{x}+k_{y} \hat{y}$, and $\hat{h}\left( \pm k_{z}\right)$ and $\hat{v}\left( \pm k_{z}\right)$ can be obtained from (1) with $\hat{k}_{s}=\left(k_{x} \hat{x}+k_{y} \hat{y} \pm k_{z} \hat{z}\right) / k_{0}$.

Since the surface height variations are much smaller than the wavelength $\left(\Delta \ll \lambda_{0}\right)$, the induced polarization current on the top rough layer closely resembles that of a smooth layer with the same dielectric constant and thickness $d$. Under this assumption, the polarization current may be expanded in terms of a convergent perturbation series in $\Delta$, and is given by

$$
\mathbf{J}(\mathbf{r})=\sum_{n=0}^{\infty} \mathbf{J}_{n}(\mathbf{r}) \Delta^{n}
$$

where $\mathbf{J}_{0}(\mathbf{r})$ is the induced polarization current in the unperturbed layer. Then by substituting this expansion into (6) and collecting terms of equal powers in $\Delta$, a recursive set of equations for the components of the polarization current can be obtained and is given by

$$
\begin{aligned}
& \frac{1}{\epsilon-1} \tilde{\mathbf{J}}_{0}\left(\mathbf{k}_{\perp}, z\right) \\
& =-i 4 \pi^{2} k_{0} Y_{0} \delta\left(\mathbf{k}_{\perp}-\mathbf{k}_{\perp}^{i}\right)\left[\mathbf{P}_{i} e^{-i k_{z}^{i} z}+\mathbf{P}_{r} e^{i k_{z}^{i} z}\right] \\
& \quad+k_{0}^{2} \int_{0}^{d} \tilde{\mathbf{G}}\left(\mathbf{k}_{\perp} ; z, z^{\prime}\right) \cdot \tilde{\mathbf{J}}_{0}\left(\mathbf{k}_{\perp}, z^{\prime}\right) d z^{\prime} \\
& \frac{1}{\epsilon-1} \tilde{\mathbf{J}}_{N}\left(\mathbf{k}_{\perp}, z\right) \\
& \quad k_{0}^{2} \int_{0}^{d} \tilde{\mathbf{G}}\left(\mathbf{k}_{\perp} ; z, z^{\prime}\right) \cdot \tilde{\mathbf{J}}_{N}\left(\mathbf{k}_{\perp}, z^{\prime}\right) d z^{\prime} \\
& \quad+k_{0}^{2} \tilde{\mathbf{G}}\left(\mathbf{k}_{\perp} ; z, d\right) \cdot \tilde{\mathbf{V}}_{N} .
\end{aligned}
$$

Here, $\tilde{\mathbf{V}}_{N}$ is the source function for the $N$ th-order integral equation with a closed-form representation

$$
\begin{aligned}
\tilde{\mathbf{V}}_{N}= & \sum_{n=0}^{N-1} \sum_{m=0}^{N-n-1} \frac{\left(\begin{array}{c}
N-n-1 \\
m
\end{array}\right)\left(i k_{z}\right)^{m}}{(N-n) !} \\
& \cdot\left[\frac{\partial^{N-n-m-1}}{\partial\left(z^{\prime}\right)^{N-n-m-1}} \tilde{\mathbf{J}}_{n}\left(\mathbf{k}_{\perp}, d\right)\right] * \bigotimes F\left(\mathbf{k}_{\perp}\right) .
\end{aligned}
$$

The integral equations so obtained are Fredholm integral equations of the second kind, for which analytical solutions can be obtained. Note that the solution of the zeroth-order equation is the source function for the first-order equation and the $N$ th-order equation has an excitation function which consists of $N-1$ lower order polarization currents. To solve (8), let us first split the integral into two integrals: one over the interval $[0, z]$ and the other over the interval $[z, d]$. Extending the integration limits of the second integral over the entire interval $[0, d]$ by adding and subtracting an integral over the interval $[0, z]$ and noting $\hat{h}\left(k_{z}\right)=\hat{h}\left(-k_{z}\right)$, (8) can be written as

$$
\begin{aligned}
\frac{1}{\epsilon-1} & \tilde{\mathbf{J}}_{0}\left(\mathbf{k}_{\perp}, z\right) \\
= & -i 4 \pi^{2} k_{0} Y_{0} \delta\left(\mathbf{k}_{\perp}-\mathbf{k}_{\perp}^{i}\right)\left[\mathbf{P}_{i} e^{-i k_{z}^{i} z}+\mathbf{P}_{r} e^{i k_{z}^{i} z}\right] \\
& -\hat{z} \hat{z} \cdot \tilde{\mathbf{J}}_{0}\left(\mathbf{k}_{\perp}, z\right)+\frac{i k_{0}^{2}}{2 k_{z}} \int_{0}^{z}\left\{\hat{h}\left(k_{z}\right) \hat{h}\left(k_{z}\right)\right. \\
& \cdot\left[e^{-i k_{z}\left(z^{\prime}-z\right)}-e^{i k_{z}\left(z^{\prime}-z\right)}\right]+\left[\hat{v}\left(k_{z}\right) \hat{v}\left(k_{z}\right) e^{-i k_{z}\left(z^{\prime}-z\right)}\right. \\
& \left.\left.-\hat{v}\left(-k_{z}\right) \hat{v}\left(-k_{z}\right) e^{i k_{z}\left(z^{\prime}-z\right)}\right]\right\} \cdot \tilde{\mathbf{J}}_{0}\left(\mathbf{k}_{\perp}, z^{\prime}\right) d z^{\prime} \\
& +\frac{i k_{0}^{2}}{2 k_{z}}\left\{\left[r_{h} e^{i k_{z} z}+e^{-i k_{z} z}\right] \hat{h}\left(k_{z}\right) \hat{h}\left(k_{z}\right)+\left[r_{v} e^{i k_{z} z} \hat{v}\left(k_{z}\right)\right.\right. \\
& \left.\left.+e^{-i k_{z} z} \hat{v}\left(-k_{z}\right)\right] \hat{v}\left(-k_{z}\right)\right\} \cdot \int_{0}^{d} \tilde{\mathbf{J}}_{0}\left(\mathbf{k}_{\perp}, z^{\prime}\right) d z^{\prime} .
\end{aligned}
$$

$$
\tilde{\tilde{\mathbf{G}}}\left(\mathbf{k}_{\perp} ; z, z^{\prime}\right)=-\hat{z} \hat{z} \frac{\delta\left(z-z^{\prime}\right)}{k_{0}^{2}}+\frac{i}{2 k_{z}}\left\{\begin{array}{cc}
\left\{\left[r_{h} \hat{h}\left(k_{z}\right) e^{i k_{z} z}+\hat{h}\left(-k_{z}\right) e^{-i k_{z} z}\right] \cdot \hat{h}\left(-k_{z}\right) e^{i k_{z} z^{\prime}}\right. & \\
\left.+\left[r_{v} \hat{v}\left(k_{z}\right) e^{i k_{z} z}+\hat{v}\left(-k_{z}\right) e^{-i k_{z} z}\right] \cdot \hat{v}\left(-k_{z}\right) e^{i k_{z} z^{\prime}}\right\} & z<z^{\prime} \\
\left\{\hat{h}\left(k_{z}\right)\left[r_{h} \hat{h}\left(-k_{z}\right) e^{i k_{z} z^{\prime}}+\hat{h}\left(k_{z}\right) e^{-i k_{z} z^{\prime}}\right] e^{i k_{z} z}\right. & \\
\left.+\hat{v}\left(k_{z}\right)\left[r_{v} \hat{v}\left(-k_{z}\right) e^{i k_{z} z^{\prime}}+\hat{v}\left(k_{z}\right) e^{-i k_{z} z^{\prime}}\right] e^{i k_{z} z}\right\} & z>z^{\prime}
\end{array}\right.
$$


Noting that the second integral in (10) is a constant function of $z$ and that the first integral is of convolution type in $z$, (10) is recognized as a vector Volta integral equation that can be solved analytically using the Laplace transformation or Picard's Process of successive approximation [15]. Since the involved integral in (10) is explicit in terms of variable $\mathbf{k}_{\perp}$, it can be shown that $\tilde{\mathbf{J}}_{0}\left(\mathbf{k}_{\perp}, z\right)$ is of the form

$$
\tilde{\mathbf{J}}_{0}\left(\mathbf{k}_{\perp}, z\right)=(2 \pi)^{2} \delta\left(\mathbf{k}_{\perp}-\mathbf{k}_{\perp}^{i}\right) \tilde{\mathbf{J}}_{0}(z)
$$

The polarization current can be decomposed into its principal components given by

$$
\tilde{\mathbf{J}}_{0}(z)=J_{0 h}(z) \hat{h}\left(k_{z}^{i}\right)+J_{0 t}(z) \hat{t}\left(k_{z}^{i}\right)+J_{0 z}(z) \hat{z}
$$

where $\hat{t}\left(k_{z}^{i}\right)=\hat{z} \times \hat{h}\left(k_{z}^{i}\right)$. Evaluating the inner product of (10) with $\hat{h}\left(k_{z}^{i}\right), \hat{t}\left(k_{z}^{i}\right)$, and $\hat{z}$, three uncoupled scalar Volta integral equations are obtained. Solutions to the resulted integral equations for the three components of current are of the following form:

$$
\begin{aligned}
& J_{0 h}(z)=A_{h}^{0} e^{i k_{1 z}^{i} z}+B_{h}^{0} e^{-i k_{1 z}^{i} z} \\
& J_{0 t}(z)=A_{v}^{0} e^{i k_{1 z}^{i} z}+B_{v}^{0} e^{-i k_{1 z}^{i} z} \\
& J_{0 z}(z)=-\frac{k_{\rho}^{i}}{k_{1 z}}\left\{A_{v}^{0} e^{i k_{1 z}^{i} z}-B_{v}^{0} e^{-i k_{1 z}^{i} z}\right\}
\end{aligned}
$$

After a long algebraic manipulation, closed-form expressions for the zeroth-order polarization current are obtained

$$
\begin{aligned}
J_{0 h}(z)= & -i \frac{2 k_{0} k_{z}^{i}}{k_{z}^{i}+k_{1 z}^{i}} Y_{0}(\epsilon-1) C_{0}^{h}\left(\mathbf{k}^{i}, z\right)\left[\mathbf{P}_{i} \cdot \hat{h}\left(k_{z}^{i}\right)\right] \\
& \cdot e^{-i k_{z}^{i} d} \\
J_{0 t}(z)= & -i \frac{2 k_{0} k_{z}^{i} k_{1 z}^{i}}{k_{\rho}^{i}\left(\epsilon k_{z}^{i}+k_{1 z}^{i}\right)} Y_{0}(\epsilon-1) C_{0}^{v}\left(\mathbf{k}^{i}, z\right)\left[\mathbf{P}_{i} \cdot \hat{z}\right] \\
& \cdot e^{-i k_{z}^{i} d} \\
J_{0 z}(z)= & -i \frac{2 k_{0} k_{z}^{i}}{\epsilon k_{z}^{i}+k_{1 z}^{i}} Y_{0}(\epsilon-1) C_{1}^{v}\left(\mathbf{k}^{i}, z\right)\left[\mathbf{P}_{i} \cdot \hat{z}\right] e^{-i k_{z}^{i} d}
\end{aligned}
$$

where

$$
\begin{aligned}
k_{1 z}^{i} & =k_{0} \sqrt{\epsilon-\sin ^{2} \theta_{i}} \\
k_{\rho}^{i} & =k_{0} \sin \theta_{i} \\
R_{h}^{i} & =\frac{k_{z}^{i}-k_{1 z}^{i}}{k_{z}^{i}+k_{1 z}^{i}} \\
R_{v}^{i} & =\frac{\epsilon k_{z}^{i}-k_{1 z}^{i}}{\epsilon k_{z}^{i}+k_{1 z}^{i}} \\
C_{n}^{h}(\mathbf{k}, z) & =\frac{(-1)^{n}\left(R_{h}-r_{h}\right) e^{i k_{1 z} z}+\left(R_{h} r_{h}-1\right) e^{-i k_{1 z} z}}{R_{h}\left(R_{h}-r_{h}\right) e^{i k_{1 z} d}+\left(R_{h} r_{h}-1\right) e^{-i k_{1 z} d}} \\
C_{n}^{v}(\mathbf{k}, z) & =\frac{(-1)^{n}\left(r_{v}-R_{v}\right) e^{i k_{1 z} z}+\left(R_{v} r_{v}-1\right) e^{-i k_{1 z} z}}{R_{v}\left(R_{v}-r_{v}\right) e^{i k_{1 z} d}+\left(R_{v} r_{v}-1\right) e^{-i k_{1 z} d}} .
\end{aligned}
$$

The source function of (9) can be written as

$$
k_{0}^{2} \tilde{\tilde{\mathbf{G}}}\left(\mathbf{k}_{\perp} ; z, d\right) \cdot \tilde{\mathbf{V}}_{N} \triangleq-i k_{0} Y_{0}\left(\mathbf{q}_{N i} e^{-i k_{z} z}+\mathbf{q}_{N r} e^{i k_{z} z}\right)
$$

where

$$
\begin{aligned}
\mathbf{q}_{N i}= & \frac{-Z_{0} k_{0}}{2 k_{z}} e^{i k_{z} d} \cdot\left\{\left[\hat{h}\left(k_{z}\right) \cdot \tilde{\mathbf{V}}_{N}\right] \hat{h}\left(k_{z}\right)\right. \\
& \left.+\left[\hat{v}\left(-k_{z}\right) \cdot \tilde{\mathbf{V}}_{N}\right] \hat{v}\left(-k_{z}\right)\right\} \\
\mathbf{q}_{N r}= & \frac{-Z_{0} k_{0}}{2 k_{z}} e^{i k_{z} d} \cdot\left\{\left[\hat{h}\left(k_{z}\right) \cdot \tilde{\mathbf{V}}_{N}\right] \hat{h}\left(k_{z}\right)\right. \\
& \left.+\left[\hat{v}\left(-k_{z}\right) \cdot \tilde{\mathbf{V}}_{N}\right] \hat{v}\left(k_{z}\right)\right\} .
\end{aligned}
$$

Noted that the vector integral equation (9) and the source function for the $N$ th-order polarization current are identical to those of the zeroth-order polarization current and, therefore, a similar solution can be easily obtained. By decomposing the $N$ th-order polarization current in terms of its three principle components, it can be shown that

$$
\begin{aligned}
& \tilde{J}_{N h}\left(\mathbf{k}_{\perp}, z\right)=\frac{i k_{0}^{2}(\epsilon-1)}{k_{z}+k_{1 z}} C_{0}^{h}(\mathbf{k}, z)\left[\tilde{\mathbf{V}}_{N} \cdot \hat{h}\left(k_{z}\right)\right] \\
& \tilde{J}_{N t}\left(\mathbf{k}_{\perp}, z\right)=\frac{i k_{0} k_{1 z}(\epsilon-1)}{\epsilon k_{z}+k_{1 z}} C_{0}^{v}(\mathbf{k}, z)\left[\tilde{\mathbf{V}}_{N} \cdot \hat{v}\left(-k_{z}\right)\right] \\
& \tilde{J}_{N z}\left(\mathbf{k}_{\perp}, z\right)=\frac{i k_{0} k_{\rho}(\epsilon-1)}{\epsilon k_{z}+k_{1 z}} C_{1}^{v}(\mathbf{k}, z)\left[\tilde{\mathbf{V}}_{N} \cdot \hat{v}\left(-k_{z}\right)\right]
\end{aligned}
$$

\section{A. Scattering Coefficients}

Once the polarization current is obtained, the scattered field in region $z>d$ can be obtained from (3). Assuming that the surface perturbation is localized and the observation point $\mathrm{r}=r\left(\sin \theta_{s} \cos \phi_{s} \hat{x}+\sin \theta_{s} \sin \phi_{s} \hat{y}+\cos \theta_{s} \hat{z}\right)$ is far from the scatterer, the far-field approximation can be used to find the scattered fields. Using the stationary phase approximation in the far-field region, the Green's function is reduced to

$$
\begin{aligned}
\overline{\overline{\mathbf{G}}}\left(\mathbf{r}, \mathbf{r}^{\prime}\right)= & \frac{e^{i k_{0} r}}{4 \pi r}\left\{\left[\hat{h}\left(k_{z}^{s}\right) \hat{h}\left(k_{z}^{s}\right) r_{h}^{s}+\hat{v}\left(k_{z}^{s}\right) \hat{v}\left(-k_{z}^{s}\right) r_{v}^{s}\right] e^{-i \mathbf{K}^{s} r^{\prime}}\right. \\
& \left.+\left[\hat{h}\left(k_{z}^{s}\right) \hat{h}\left(k_{z}^{s}\right)+\hat{v}\left(k_{z}^{s}\right) \hat{v}\left(k_{z}^{s}\right)\right] e^{-i \mathbf{k}^{s} r^{\prime}}\right\} .
\end{aligned}
$$

Substituting (13) and the polarization currents into (3) and expanding the integral similar to those used in (5), the $N$ thorder scattered field is given by a power series in $\Delta f(x, y)$ [similar to (3)]. In this process, the $N$ th-order scattered field components are found to be

$$
\begin{aligned}
& \mathbf{E}_{N}^{s}(\mathbf{r}) \cdot \hat{h}\left(k_{z}^{s}\right)=i k_{0} Z_{0} \Delta^{N} \frac{e^{i k_{0} r}}{4 \pi r} e^{-i k_{z}^{s} d} \sum_{n=0}^{N-1} \sum_{m=0}^{N-n-1} \\
& \cdot \frac{\left(\begin{array}{c}
N-n-1 \\
m
\end{array}\right)\left(i k_{z}^{s}\right)^{m}}{(N-n) !}\left[R_{h}^{s}+(-1)^{m}\right] \\
& \cdot C_{m}^{h}\left(\mathbf{k}^{s}, d\right)\left[\frac{\partial^{N-n-m-1}}{\partial\left(z^{\prime}\right)^{N-n-m-1}} \tilde{\mathbf{J}}_{n}\left(\mathbf{k}_{\perp}^{s}, d\right)\right] \\
& * \bigotimes^{N-n} F\left(\mathbf{k}_{\perp}^{s}\right) \cdot \hat{h}\left(k_{z}^{s}\right)
\end{aligned}
$$




$$
\begin{aligned}
\mathbf{E}_{N}^{s}(\mathbf{r}) \cdot \hat{v}\left(k_{z}^{s}\right)= & i k_{0} Z_{0} \Delta^{N} \frac{e^{i k_{0} r}}{4 \pi r} e^{-i k_{z}^{s} d} \sum_{n=0}^{N-1} \sum_{m=0}^{N-n-1} \\
& \cdot \frac{\left(\begin{array}{c}
N-n-1 \\
m
\end{array}\right)\left(i k_{z}^{s}\right)^{m}}{(N-n) !}\left\{\left[R_{v}^{s}-(-1)^{m}\right]\right. \\
& \cdot C_{m}^{v}\left(\mathbf{k}^{s}, d\right) \cos \theta_{s} \hat{t}\left(k_{z}^{s}\right) \\
& \left.+\left[R_{v}^{s}+(-1)^{m}\right] C_{m+1}^{v}\left(\mathbf{k}^{s}, d\right) \sin \theta_{s} \hat{z}\right\} \\
& \cdot\left[\frac{\partial^{N-n-m-1}}{\partial\left(z^{\prime}\right)^{N-n-m-1}} \tilde{\mathbf{J}}_{n}\left(\mathbf{k}_{\perp}^{s}, d\right)\right] \\
& * \bigotimes^{N-n} F\left(\mathbf{k}_{\perp}^{s}\right) .
\end{aligned}
$$

The polarimetric response of a target can be obtained from its complex scattering matrix, defined by

$$
\mathbf{E}^{s}=\frac{e^{i k r}}{r} \overline{\overline{\mathbf{S}}} \mathbf{E}^{i}
$$

The elements of the bistatic scattering matrix can simply be computed by setting $\mathbf{P}_{i}=\hat{h}\left(k_{z}^{i}\right)$ and $\mathbf{P}_{i}=\hat{v}\left(k_{z}^{i}\right)$ in (14) and (15). For distributed targets, such as rough surfaces, the quantities of interest are the elements of the differential covariance matrix, defined by

$$
\sigma_{i j p q}^{0}=\lim _{A \rightarrow \infty} \frac{4 \pi}{A}\left\langle S_{i j} S_{p q}^{*}\right\rangle, \quad i, j, p, q \in\{h, v\} .
$$

Here, $\langle\cdot\rangle$ denotes ensemble averaging. These elements are in general complex quantities, except when $i=p$ and $j=q$, in which case the elements are the usual scattering coefficients. In the perturbation analysis, each element of the scattering matrix can be evaluated up to the $N$ th-order, that is

$$
S_{i j}=\sum_{n=1}^{N} S_{i j}^{(n)} \Delta^{n}, \quad i, j \in\{h, v\} .
$$

It turns out that simple expressions for the first-order elements can be obtained and are given by

$$
\begin{aligned}
S_{h h}^{(1)}= & \frac{k_{0}^{2} k_{z}^{i} k_{z}^{s}(\epsilon-1) e^{-i\left(k_{z}^{i}+k_{z}^{s}\right) d}}{\pi\left(k_{z}^{i}+k_{1 z}^{i}\right)\left(k_{z}^{s}+k_{1 z}^{s}\right)} C_{0}^{h}\left(\mathbf{k}^{s}, d\right) C_{0}^{h}\left(\mathbf{k}^{i}, d\right) \\
& \cdot \cos \left(\phi_{s}-\phi_{i}\right) F\left(\mathbf{k}_{\perp}^{s}-\mathbf{k}_{\perp}^{i}\right) \\
S_{h v}^{(1)}= & \frac{k_{0} k_{z}^{i} k_{z}^{s} k_{1 z}^{i}(\epsilon-1) e^{-i\left(k_{z}^{i}+k_{z}^{s}\right) d}}{\pi\left(\epsilon k_{z}^{i}+k_{1 z}^{i}\right)\left(k_{z}^{s}+k_{1 z}^{s}\right)} C_{0}^{h}\left(\mathbf{k}^{s}, d\right) C_{0}^{v}\left(\mathbf{k}^{i}, d\right) \\
& \cdot \sin \left(\phi_{s}-\phi_{i}\right) F\left(\mathbf{k}_{\perp}^{s}-\mathbf{k}_{\perp}^{i}\right) \\
S_{v h}^{(1)}= & \frac{k_{0} k_{z}^{i} k_{z}^{s} k_{1 z}^{s}(\epsilon-1) e^{-i\left(k_{z}^{i}+k_{z}^{s}\right) d}}{\pi\left(\epsilon k_{z}^{s}+k_{1 z}^{s}\right)\left(k_{z}^{i}+k_{1 z}^{i}\right)} C_{0}^{h}\left(\mathbf{k}^{i}, d\right) C_{0}^{v}\left(\mathbf{k}^{s}, d\right) \\
& \cdot \sin \left(\phi_{s}-\phi_{i}\right) F\left(\mathbf{k}_{\perp}^{s}-\mathbf{k}_{\perp}^{i}\right) \\
S_{v v}^{(1)}= & \frac{k_{z}^{i} k_{1 z}^{i}(\epsilon-1) e^{-i\left(k_{z}^{i}+k_{z}^{s}\right) d}}{\pi\left(\epsilon k_{z}^{i}+k_{1 z}^{i}\right)\left(\epsilon k_{z}^{s}+k_{1 z}^{s}\right)} \\
& \cdot\left[-k_{z}^{s} k_{1 z}^{s} C_{0}^{v}\left(\mathbf{k}^{s}, d\right) C_{0}^{v}\left(\mathbf{k}^{i}, d\right) \cos \left(\phi_{s}-\phi_{i}\right)\right. \\
& \left.\quad+\frac{\epsilon k_{z}^{s} k_{\rho}^{s} k_{\rho}^{i}}{k_{1 z}^{i}} \cdot C_{1}^{v}\left(\mathbf{k}^{s}, d\right) C_{1}^{v}\left(\mathbf{k}^{i}, d\right)\right] F\left(\mathbf{k}_{\perp}^{s}-\mathbf{k}_{\perp}^{i}\right) .
\end{aligned}
$$

In these expressions $F\left(\mathbf{k}_{\perp}^{s}-\mathbf{k}_{\perp}^{i}\right)$ is the only indeterministic factor and, therefore, the elements of the differential covariance matrix can easily be obtained by noting that

$$
\lim _{A \rightarrow \infty} \frac{1}{A}\left\langle\left|\Delta F\left(\mathbf{k}_{\perp}^{s}-\mathbf{k}_{\perp}^{i}\right)\right|^{2}\right\rangle=W\left(\mathbf{k}_{\perp}^{s}-\mathbf{k}_{\perp}^{i}\right)
$$

where $W\left(\mathbf{k}_{\perp}\right)$ is the power spectral density of the surface.

To examine the validity of the first-order results, a special case is considered. In the case of backscattering $\left(\mathbf{k}_{\perp}^{s}=-\mathbf{k}_{\perp}^{i}\right)$ and for a homogeneous profile where $R_{v}=r_{v}$ and $R_{h}=r_{h}$, the first-order backscattering coefficients are given by

$$
\begin{aligned}
\sigma_{h h h h}^{0}= & \frac{4}{\pi} k_{0}^{4} \cos ^{4} \theta_{i}\left|R_{h}^{i}\right|^{2} W\left(-2 \mathbf{k}_{\perp}^{i}\right), \\
\sigma_{v v v v}^{0}= & \frac{4}{\pi} \cos ^{4} \theta_{i}\left|\frac{\left(k_{1}^{2}-k_{0}^{2}\right)\left(k_{1 z}^{2}+k_{1}^{2} \sin ^{2} \theta_{i}\right)}{\left(k_{1 z}^{i}+\epsilon k_{z}^{i}\right)^{2}}\right|^{2} \\
& \cdot W\left(-2 \mathbf{k}_{\perp}^{i}\right) \\
\sigma_{h v h v}^{0}= & \sigma_{v h v h}^{0} \\
= & 0
\end{aligned}
$$

which are in agreement with the results reported in the literature [5]. Before we proceed with the higher order scattering solutions, the following observations are in order. The analysis is simplified if we assume that the surface-height profile $f(x, y)$ is a Gaussian random field. There is some evidence that this assumption is reasonable for some surfaces of practical importance [1]. Since Fourier transformation is a linear operation, $F\left(\mathbf{k}_{\perp}\right)$ is also Gaussian. It is well known that the following identities hold for a zero-mean jointly Gaussian random vector $\left\{X_{1}, \cdots, X_{n}\right\}$ :

$$
\begin{aligned}
\left\langle X_{i} X_{j} X_{k}\right\rangle= & 0 \\
\left\langle X_{i} X_{j} X_{k} X_{l}\right\rangle= & \left\langle X_{i} X_{j}\right\rangle\left\langle X_{k} X_{l}\right\rangle+\left\langle X_{i} X_{k}\right\rangle\left\langle X_{j} X_{l}\right\rangle \\
& +\left\langle X_{i} X_{l}\right\rangle\left\langle X_{j} X_{k}\right\rangle .
\end{aligned}
$$

On the other hand, it can be shown that

$$
\begin{aligned}
S_{i j}^{(1)} & \propto F\left(\mathbf{k}_{\perp}^{s}-\mathbf{k}_{\perp}^{i}\right) \\
S_{i j}^{(2)} & \propto \int_{-\infty}^{\infty} d \mathbf{k}_{\perp} F\left(\mathbf{k}_{\perp}^{s}-\mathbf{k}_{\perp}\right) F\left(\mathbf{k}_{\perp}-\mathbf{k}_{\perp}^{i}\right) I_{i j}^{(2)}\left(\mathbf{k}_{\perp}\right) \\
S_{i j}^{(3)} & \propto \iint_{-\infty}^{\infty} d \mathbf{k}_{\perp} d \mathbf{k}_{\perp}^{\prime} F\left(\mathbf{k}_{\perp}^{s}-\mathbf{k}_{\perp}\right) \\
& \cdot F\left(\mathbf{k}_{\perp}-\mathbf{k}_{\perp}^{\prime}\right) F\left(\mathbf{k}_{\perp}^{\prime}-\mathbf{k}_{\perp}^{i}\right) I_{i j}^{(3)}\left(\mathbf{k}_{\perp}, \mathbf{k}_{\perp}^{\prime}\right)
\end{aligned}
$$

where $I_{i j}^{(2)}$ and $I_{i j}^{(3)}$ are functions of polarization currents. For the evaluation of the covariance matrix, we confine our interest in perturbation terms up to $\Delta^{4}$. Substituting (25)-(27) in (17) and then using (23), the elements of covariance matrix simplify to

$$
\begin{aligned}
\left\langle S_{i j} S_{p q}^{*}\right\rangle \approx & \left\langle S_{i j}^{(1)} S_{p q}^{(1) *}\right\rangle \Delta^{2}+\left[\left\langle S_{i j}^{(2)} S_{p q}^{(2) *}\right\rangle\right. \\
& \left.+\left\langle S_{i j}^{(1)} S_{p q}^{(3) *}\right\rangle+\left\langle S_{i j}^{(3)} S_{p q}^{(1) *}\right\rangle\right] \Delta^{4} .
\end{aligned}
$$

Noting that property (23) is valid for any odd number of random variables, the elements of covariance matrix are only functions of even power of $\Delta$. Therefore, the next higher order of approximation in calculation of $\left\langle S_{i j} S_{p q}^{*}\right\rangle$ can be obtained 
by inclusion of products of the first and the fifth, the second and the fourth, and the third-order scattering terms. However, evaluation of high-order scattered fields such as fourth and fifth order are rather complex and tedious. Noting that $\Delta$ is a small quantity compared to the wavelength, the benefit of inclusion of $\Delta^{6}$ term is not significant. This argument cannot be used for the second-order solution $\left(\Delta^{4}\right.$ term) since this term is the dominant factor for some important scattering parameters such as cross-polarized backscattering coefficient and copolarized degree of correlation.

The scattering matrix elements up to third order are derived. These expressions are very lengthy and are not included in this paper. Interested readers are referred to [11]. Using these expressions in (28), the elements of the covariance matrix can be obtained. The ensemble averaging process can be carried out easily using (24) and

$$
\begin{aligned}
\Delta^{2}\left\langle F\left(\mathbf{k}_{\perp}\right) F^{*}\left(\mathbf{k}_{\perp}^{\prime}\right)\right\rangle & =\Delta^{2}\left\langle F\left(\mathbf{k}_{\perp}\right) F\left(-\mathbf{k}_{\perp}^{\prime}\right)\right\rangle \\
& =(2 \pi)^{2} \delta\left(\mathbf{k}_{\perp}-\mathbf{k}_{\perp}^{\prime}\right) W\left(\mathbf{k}_{\perp}\right) .
\end{aligned}
$$

Using the above mentioned properties, and noting that in backscatter direction $\left(\phi_{s}=\phi_{i}+\pi, \theta_{s}=\theta_{i}\right) S_{h v}^{(1)}=S_{v h}^{(1)}=0$, the cross-polarized backscattering coefficients can be obtained and are given by

$$
\begin{aligned}
\sigma_{h v h v}^{0}= & \sigma_{v h v h}^{0} \\
= & \frac{\left|k_{0} k_{z}^{i}(\epsilon-1)^{2}\right|^{2}}{16 \pi^{3}} \\
& \cdot\left|\left(1-R_{v}^{i}\right)\left(1+R_{h}^{i}\right) C_{0}^{h}\left(\mathbf{k}^{i}, d\right) C_{0}^{v}\left(\mathbf{k}^{i}, d\right)\right|^{2} \\
& \cdot \int_{-\infty}^{\infty} W\left(\mathbf{k}_{\perp}-\mathbf{k}_{\perp}^{i}\right) W\left(\mathbf{k}_{\perp}+\mathbf{k}_{\perp}^{i}\right) \\
& \cdot \sin ^{2}\left(\phi-\phi_{i}\right) \cos ^{2}\left(\phi-\phi_{i}\right) \\
& \cdot\left|\frac{k_{0}^{2}}{k_{z}+k_{1 z}} C_{0}^{h}(\mathbf{k}, d)-\frac{k_{z} k_{1 z}}{k_{1 z}+\epsilon k_{z}} C_{0}^{v}(\mathbf{k}, d)\right|^{2} d \mathbf{k}_{\perp}
\end{aligned}
$$

which satisfies the reciprocity condition. To examine the validity of (30), a homogeneous profile is considered having $R_{h}=r_{h}$ and $R_{v}=r_{v}$. In this case

$$
\begin{aligned}
\sigma_{h v h v}^{0}= & \sigma_{v h v h}^{0} \\
= & \frac{2}{\pi^{3}}\left|\frac{k_{0}\left(k_{z}^{i}\right)^{2} k_{1 z}^{i}\left(k_{1}^{2}-k_{0}^{2}\right)^{2}}{\left(k_{0}^{2} k_{1 z}^{i}+k_{1}^{2} k_{z}^{i}\right)\left(k_{z}^{i}+k_{1 z}^{i}\right)}\right|^{2} \\
& \cdot \int\left|\frac{\left(k_{x}^{i} k_{y}-k_{y}^{i} k_{x}\right)\left(k_{x}^{i} k_{x}+k_{y}^{i} k_{y}\right)}{\left(k_{\rho}^{i}\right)^{2}\left(k_{0}^{2} k_{1 z}+k_{1}^{2} k_{z}\right)}\right|^{2} \\
& \cdot W\left(\mathbf{k}_{\perp}-\mathbf{k}_{\perp}^{i}\right) W\left(\mathbf{k}_{\perp}+\mathbf{k}_{\perp}^{i}\right) d \mathbf{k}_{\perp}
\end{aligned}
$$

which is in agreement with result reported in [5].

\section{B. Phase Statistics}

Traditionally, scattering models for rough surfaces provide formulations for copolarized and cross-polarized scattering coefficients. With the advances in the development of polarimetric radar, the statistics of the phase difference of scattering matrix elements can be measured and used in inversion algorithms to retrieve the target parameters. In a polarimetric backscatter measurement, apart from the backscattering coefficients, the copolarized and cross-polarized phase differences, defined by $\phi_{c}=\phi_{h h}-\phi_{v v}$ and $\phi_{x}=\phi_{h v}-\phi_{v v}$, are two additional independent parameters, which can be used in an inversion process. In a recent paper [12], it was shown that the statistics of the phase difference can be derived from the elements of the target covariance matrix $\left(\left\langle S_{i j} S_{p q}^{*}\right\rangle\right)$ and that the probability density function (pdf) of each phasedifference can be fully determined in terms of two parameters: 1) coherent phase difference $(\zeta)$ and 2 ) degree of correlation $(\alpha)$. The coherent phase difference is the phase difference at which the pdf assumes its maximum. The degree of correlation is a real number that can vary from zero to one and is proportional to the spread of the pdf around $\zeta$, where $\alpha=0$ corresponds to a uniform distribution and $\alpha=1$ corresponds to a delta function. In terms of covariance matrix elements, $\zeta$ and $\alpha$ are given by

$$
\begin{aligned}
\zeta & =\tan ^{-1} \frac{\operatorname{Im}\left[\left\langle S_{i j} S_{v v}^{*}\right\rangle\right]}{\operatorname{Re}\left[\left\langle S_{i j} S_{v v}^{*}\right\rangle\right]} \\
\alpha & =\sqrt{\frac{\left|\left\langle S_{i j} S_{v v}^{*}\right\rangle\right|^{2}}{\left\langle\left|S_{i j}\right|^{2}\right\rangle\left\langle\left|S_{v v}\right|^{2}\right\rangle}}
\end{aligned}
$$

where subscript $i j=h h$ for copolarized and $i j=v h$ or $h v$ for cross-polarized phase difference, respectively. Referring to (18)-(21), it can easily be shown that $\alpha_{c}=1$ and $\alpha_{x}=0$ for the first-order scattering solution. Hence, $\alpha_{c}$ and $\alpha_{x}$ do not contain any information about the surface power spectral density or the surface dielectric constant. Noting that to the first-order solutions, elements of the covariance matrix are linearly proportional to the power spectral density, $\zeta_{c}$ is only a function of the surface dielectric profile.

To characterize the dependency of $\alpha_{c}$ and $\alpha_{x}$ on the surface power spectral density, we have to resort to the second-order scattering solution. Combining the first-order solution given by (18)-(21) and the second-order and third-order solutions, closed-form expressions for the parameters of phase-difference statistics can be obtained. It is found that $\alpha_{x}$ vanishes when the surface power spectral density is azimuthally symmetric; that is, if $W\left(k_{x}, k_{y}\right)=W\left(\sqrt{k_{x}^{2}+k_{y}^{2}}\right)$. This is usually the case for most practical situations, which implies the copolarized and cross-polarized backscattered fields are mutually uncorrelated.

\section{DATA Simulation AND EXPERIMENTAL RESUltS}

In the previous section, an analytical model for predicting polarimetric scattering behavior of inhomogeneous rough surfaces based on a perturbation expression of induced polarization current was obtained. Here, data simulation based on the complete second-order analytical model is carried out to investigate the sensitivity of the radar backscatter measurements to physical parameters of the surface such as the surface dielectric profile and surface power spectral density. Also, polarimetric backscatter measurements were conducted to examine the significance of the second-order solution on the overall backscatter response as a function of surface parameters and radar attributes. 


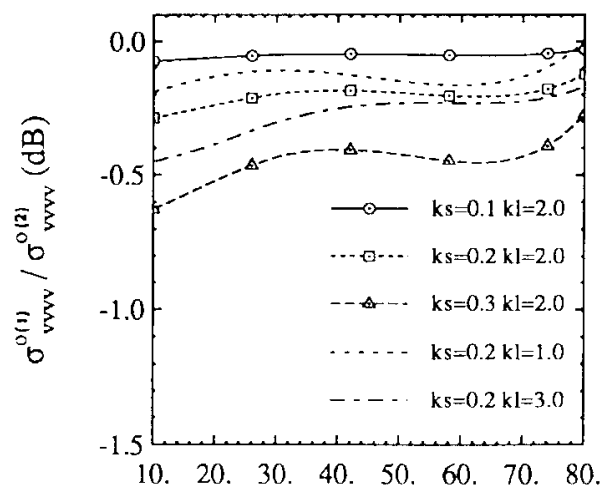

Incidence Angle (degree)

(a)

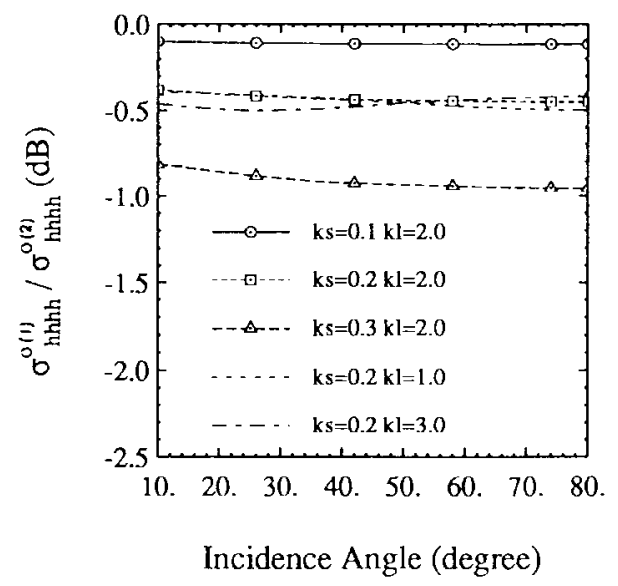

(b)

Fig. 2. Ratio of the first-order to the complete second-order solution of a homogeneous rough surface with $m_{v}=0.2(\epsilon=8.0+i 2.51$ at $1.25 \mathrm{GHz})$ as a function of incidence angle for different values of $k s$ and $k l$.

Fig. 2(a) and (b) demonstrates the significance of the second-order solution where the ratio of the first-order to the complete copolarized second-order solutions $\left.\left[\sigma^{0(1)}\right) / \sigma^{0(2)}\right]$ are plotted versus incidence angle. An exponential correlation function given by

$$
\rho(x, y)=s^{2} \exp \left[-\frac{\sqrt{x^{2}+y^{2}}}{l}\right]
$$

where $s$ is the rms height and $l$ is the surface correlation length, is used in these simulations. In Fig. 2(a) and (b), $k s$ and $k l$ are varied as free parameters and the soil surface is assumed to be a homogeneous medium with $\epsilon=8.0+i 2.51$. This dielectric constant corresponds to a moist soil surface with volumetric moisture content $m_{v}=0.2$ and is computed using the empirical formula given in [13] at $1.25 \mathrm{GHz}$ with $S=0.1$ and $C=0.3$. It is shown that the second-order scattering term is more sensitive to variations in rms height $(s)$ than it is to the surface correlation length $(l)$. The sensitivity to $s$ is higher at lower angles of incidence for $\sigma_{v v v v}^{0}$ unlike $\sigma_{h h h h}^{0}$. Fig. 3(a) and (b) shows the ratio of the first-order to the complete copolarized second-order solutions of the homogeneous rough surface as a function of soil moisture at $\theta=45^{\circ}$. Here it is shown that as the soil moisture increases

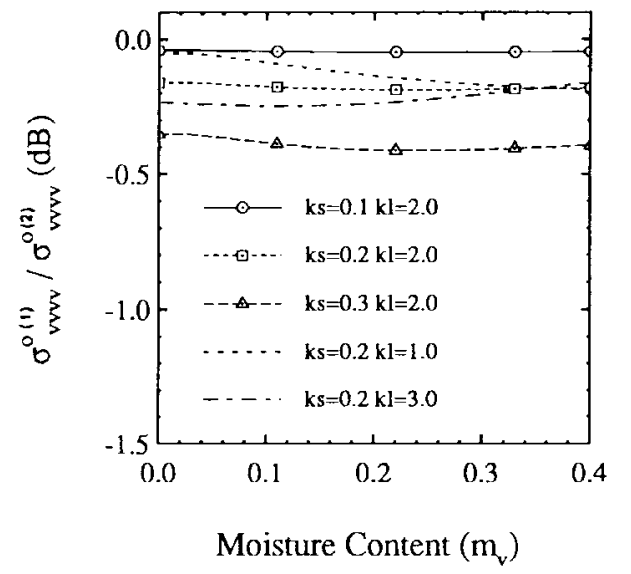

(a)

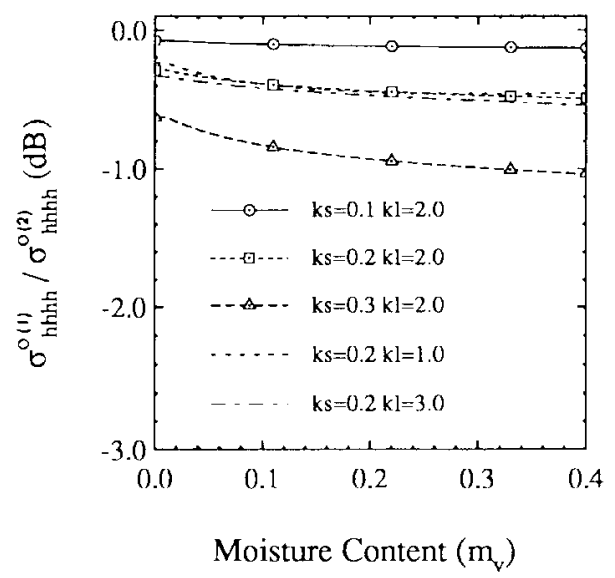

(b)

Fig. 3. Ratio of the first-order to the complete second-order solution of a homogeneous rough surface as a function of moisture content for different values of $k s$ and $k l$ at $\theta=45^{\circ}$.

from $0.01(\epsilon=2.21+i 0.002)$ to $0.4(\epsilon=14.68+i 7.5)$, the contribution from the second-order scattering term to the overall backscattering increases slightly. This effect is more pronounced for $\sigma_{h h h h}^{0}$. Figs. 2 and 3 demonstrate that the inclusion of the second-order solution is more important for calculation of $\sigma_{h h h}^{0}$ than for $\sigma_{v v v v}^{0}$. Figs. 4 and 5 show the copolarized coherent phase difference $\zeta_{c}$ calculated from the first-order and complete second-order solutions for the homogeneous surface as a function of incidence angle and soil moisture. To the first order, $\zeta_{c}$ is independent of surface roughness parameters, however, the second-order solution shows a weak dependency on $k s$ and $k l$. It is interesting to note that the sensitivity to roughness parameters disappears for incidence angles larger than $50^{\circ}$. As shown in Fig. $5, \zeta_{c}$ is relatively insensitive to moisture content for a homogeneous surface.

As mentioned before, the second-order solution is the dominant component for the cross-polarized backscattering coefficient. $\sigma_{h v h v}^{0}$ is directly proportional to the square of the rms height, thus, the dependency to $s$ is not examined. Fig. 6 shows $\sigma_{h v h v}^{0}$ of the homogeneous surface as a function of incidence angle for different values of $k l$ and $m_{v}$ while $k s=0.2$ is kept constant. Note that $\sigma_{h v h v}^{0}$ increases with increasing dielectric 


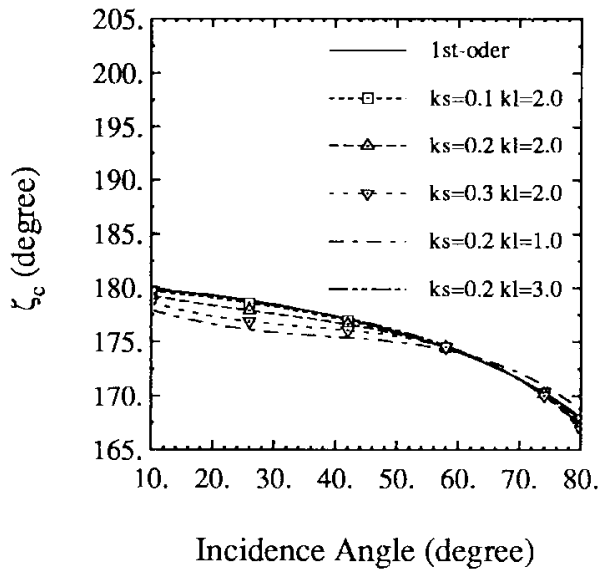

Fig. 4. Copolarized coherent phase difference of homogeneous rough surface with $m_{v}=0.2(\epsilon=8.0+i 2.51$ at $1.25 \mathrm{GHz})$ as a function of incidence angle for different values of $k s$ and $k l$.

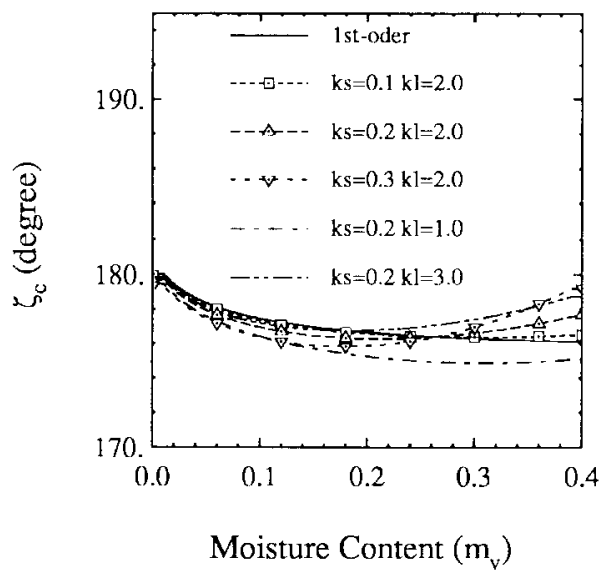

Fig. 5. Copolarized coherent phase difference of homogeneous rough surface as a function of moisture content for different values of $k s$ and $k l$ at $\theta=45^{\circ}$.

constant and decreases with increasing surface correlation length. The copolarized degree of correlation is another potential parameter that can be used in retrieval of surface physical parameters. The first-order scattering solution predict $\alpha_{c}=1$ independent of the surface physical parameters. Figs. 7 and 8 show $\alpha_{c}$ for the homogeneous rough surface as a function of incidence angle and dielectric constant for different values of $k s$ and $k l$. Note that $\alpha_{c}$, in general, has a decreasing trend with increasing incidence angle, rms height, and soil moisture. It is also noted that $\alpha_{c}$ increases when $k l$ is decreased. The total dynamic range of $\alpha_{c}$ as a function of the surface parameters is rather limited.

Next we examine the sensitivity of the polarimetric backscatter data to the surface dielectric inhomogeneity. Three dielectric profiles are considered here: 1) exponentially increasing moisture with depth; 2) exponentially decreasing moisture with depth; and 3) a two-layer step profile, as shown in Fig. 9. The exponential profiles are chosen according to [14] and are given by

$$
m_{v}(z)= \begin{cases}m_{v s}+\Delta m_{v} \frac{e^{\beta z}-1}{e^{-\beta d}-1} & -d \leq z \leq 0 \\ m_{v}(z)=m_{v}(-d) & z \leq-d\end{cases}
$$

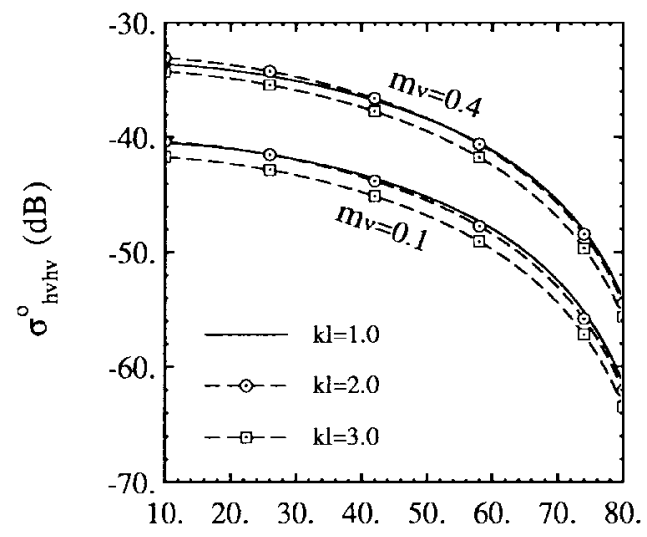

Incidence Angle (degree)

Fig. 6. Variations of cross-polarized backscattering coefficient as a function of incidence angle, moisture content $\left(\epsilon=4.89+i 0.92\right.$ for $m_{v}=0.1$ and $\epsilon=14.68+i 7.5$ for $m_{v}=0.4$ at $1.25 \mathrm{GHz}$ ) and correlation length for a surface with $k s=0.2$.

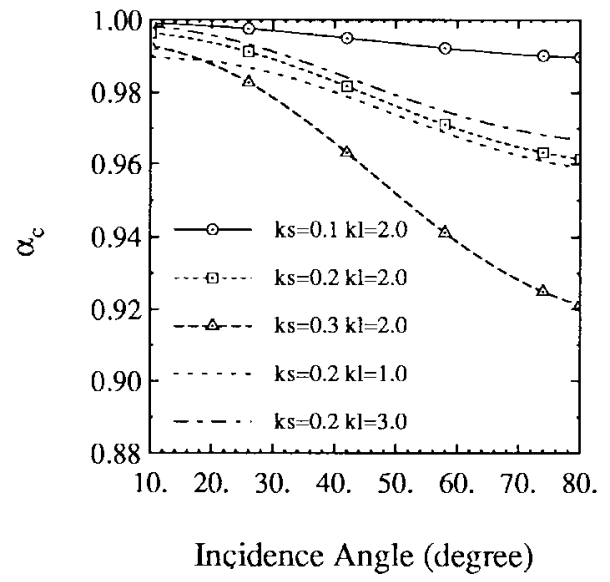

Fig. 7. Sensitivity of copolarized degree of correlation to incidence angle for different values of $k s$ and $k l$ and moisture content $m_{v}=0.2(\epsilon=8.0+i 2.51$ at $1.25 \mathrm{GHz}$ ).

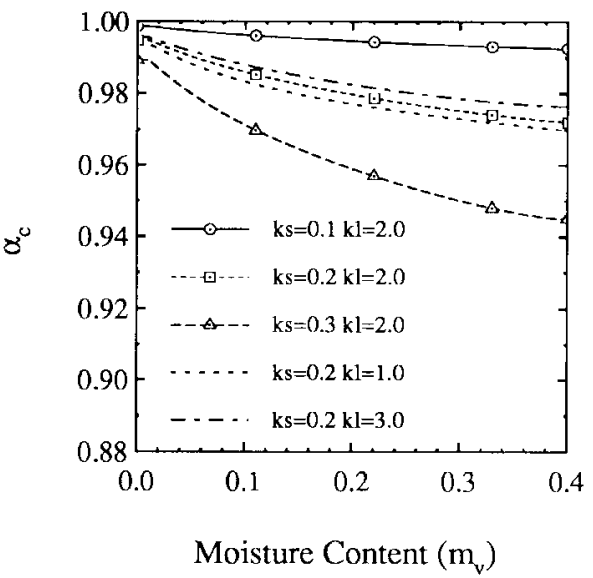

Fig. 8. Sensitivity of copolarized degree of correlation to soil moisture content for different values of $k s$ and $k l$ at $\theta=45^{\circ} . \alpha_{c}$ does not show much sensitivity to $k l$.

where $m_{v s}$ is the surface moisture content and $\Delta m_{v}$ is the increment of moisture at a depth $d$ below the surface. The moisture content below depth $d$ is considered to be uniform. In all cases the backscatter parameters are compared with a 


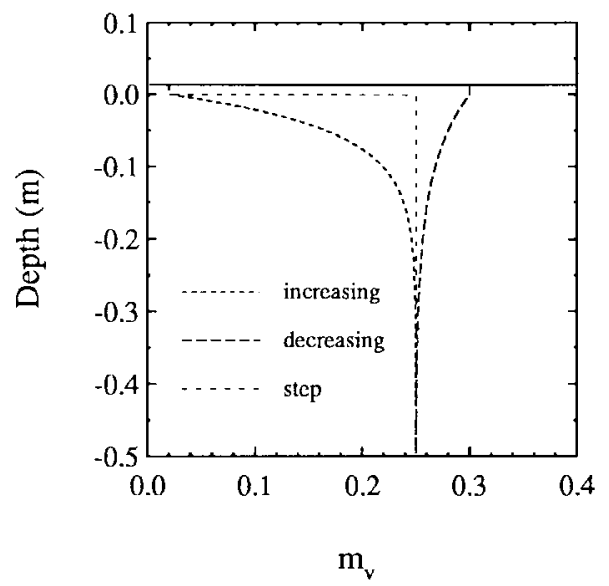

Fig. 9. Three different moisture profiles used in the backscattering simulations: increasing exponential with $\beta=20$, decreasing exponential with $\beta=10$, and step.

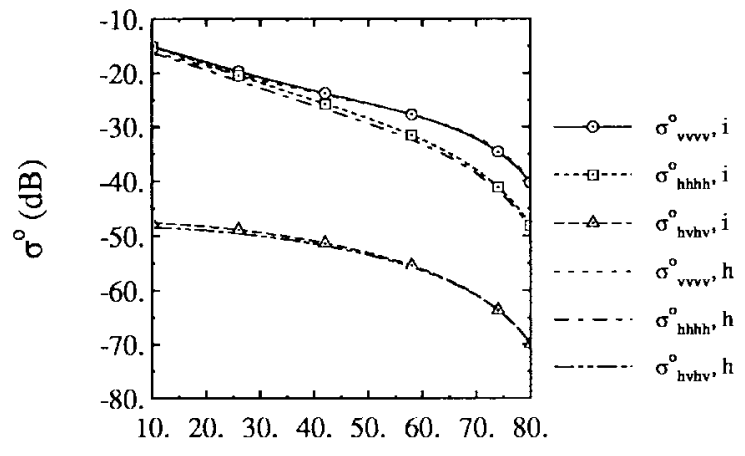

Incidence Angle (degree)

Fig. 10. Comparison of backscattering coefficients calculated for the homogeneous and increasing exponential moisture profiles for a rough surface with $k s=0.2$ and $k l=2$.

homogeneous profile having a dielectric constant equal to that of the inhomogeneous profile at the interface. Figs. 10 and 11 show the backscattering coefficients for a surface with the increasing and decreasing exponential dielectric profiles and having $k s=0.2, k l=2$. Note that the backscattering coefficients are insensitive to moisture profiles and the backscattering coefficients are basically indistinguishable from those of the homogeneous profile having the same dielectric constant as that of the inhomogeneous profile at the interface. This is due to the tapered impedance matching nature of the profile. However, this is not the case for the step profile as shown in Fig. 12. The difference in $\sigma^{\circ}$, depending on the incidence angle, can be as high as $10 \mathrm{~dB}$. The only sensitive parameter to moisture variations in depth for continuous profiles is the co-polarized coherent phase difference as is shown in Fig. 13, where $\zeta_{c}$ for the homogeneous, increasing, and step moisture profiles are shown. $\zeta_{c}$ does not show any sensitivity for decreasing profiles. It should be pointed out that the calculation of the complete second-order solution involves numerical evaluation of twofold integrals. To provide a feeling for the required computation time, the calculation of backscattering coefficients and phase difference statistics for one incidence angle would take about one minute on a Sun workstation Ultra 2.

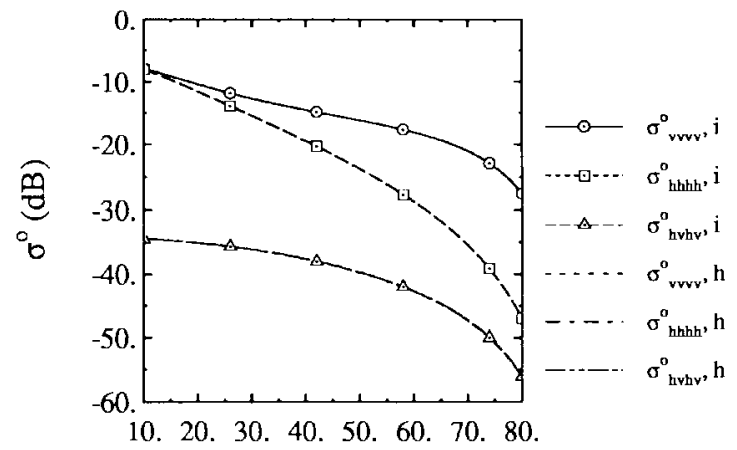

Incidence Angle (degree)

Fig. 11. Comparison of backscattering coefficients calculated for the homogeneous and decreasing exponential moisture profiles for a rough surface with $k s=0.2$ and $k l=2$.

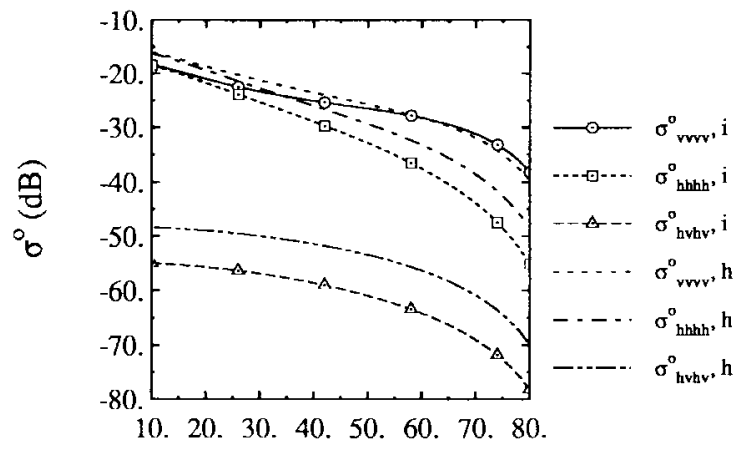

Incidence Angle (degree)

Fig. 12. Comparison of backscattering coefficients calculated for the homogeneous and step moisture profiles for a rough surface with $k s=0.2$ and $k l=2$.

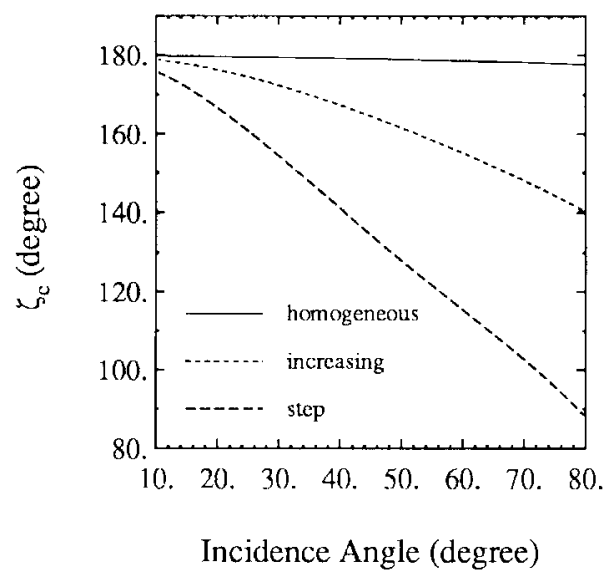

Fig. 13. Copolarized coherent phase difference calculated for the homogeneous, increasing exponential, and step moisture profiles for a rough surface with $k s=0.2$ and $k l=2$.

The validity of the analytical results are also examined by conducting backscatter measurements. The backscatter measurements were performed polarimetrically using the indoor bistatic facilities of the Radiation Laboratory, University of Michigan, Ann Arbor [16]. The backscatter data were collected from a rough layer of sand above a perfectly conducting ground plane at center frequency $9.25 \mathrm{GHz}$ with a bandwidth 


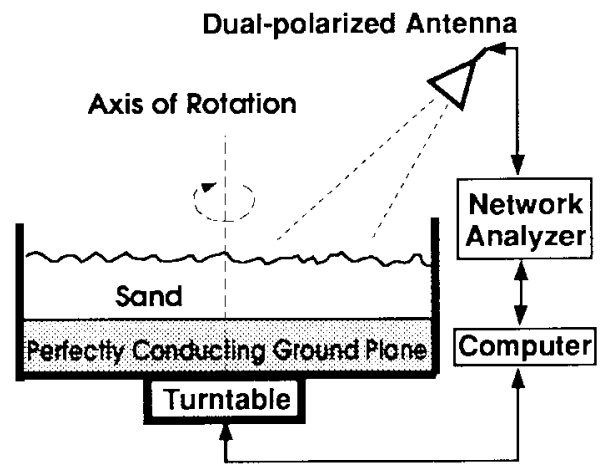

Fig. 14. Simplified block diagram of the experimental setup.

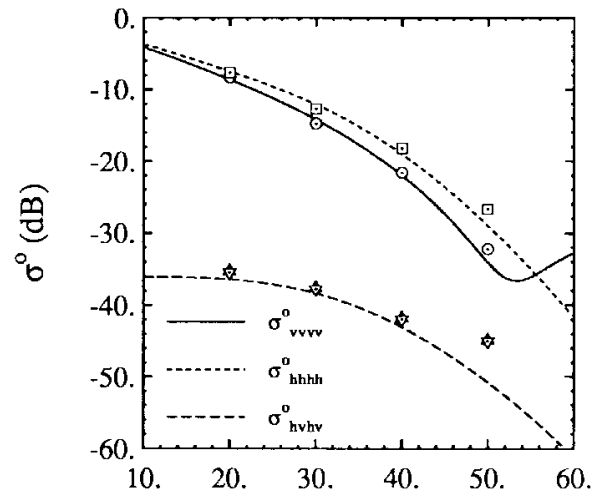

Incidence Angle (degree)

(a)

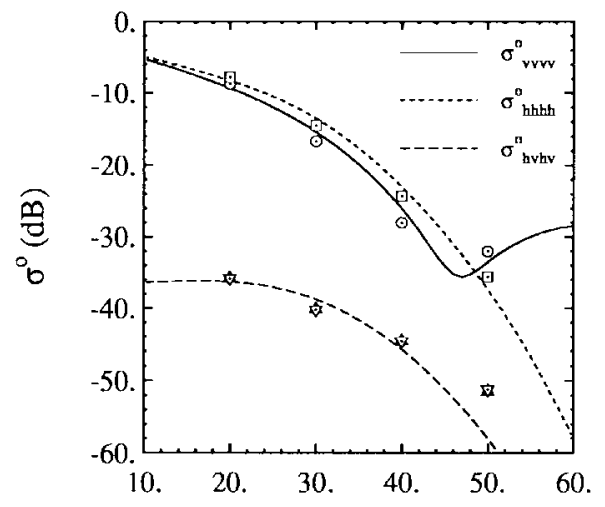

Incidence Angle (degree)

(b)

Fig. 15. Comparison of the measured and the complete second-order simulated backscatter for a sand layer of thickness (a) $2.52 \mathrm{~cm}$ and (b) $3.53 \mathrm{~cm}$ above a perfectly conducting ground plane at $9.25 \mathrm{GHz}$. Symbols represent the measured quantities and the lines are the theoretical calculations.

of $1.5 \mathrm{GHz}$. A $6^{\prime} \times 6^{\prime}$ sand-box on top of a computercontrolled turntable was used to contain the sand layer. The antenna footprint covered an area of about $0.27 \mathrm{~s} \theta \mathrm{m}^{2}$ on the sandbox and collection of independent backscatter data was facilitated by rotating the sandbox at steps of $5^{\circ}$. The wide bandwidth of the radar system was used to "range-gate" the possible unwanted radar backscatter from the sandbox walls and edges. A simplified block diagram of the measurement system is shown in Fig. 14.

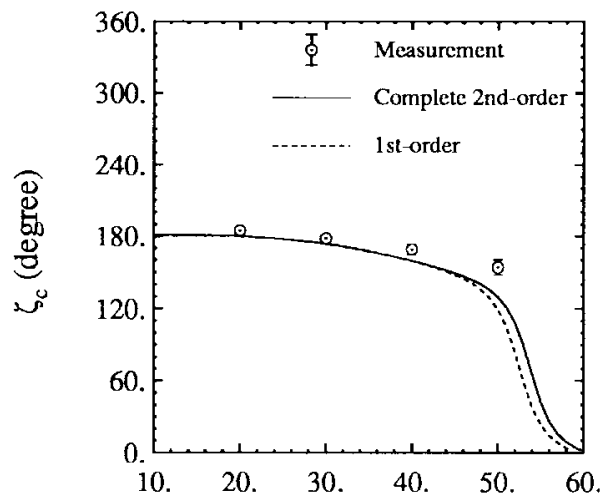

Incidence Angle (degree)

(a)

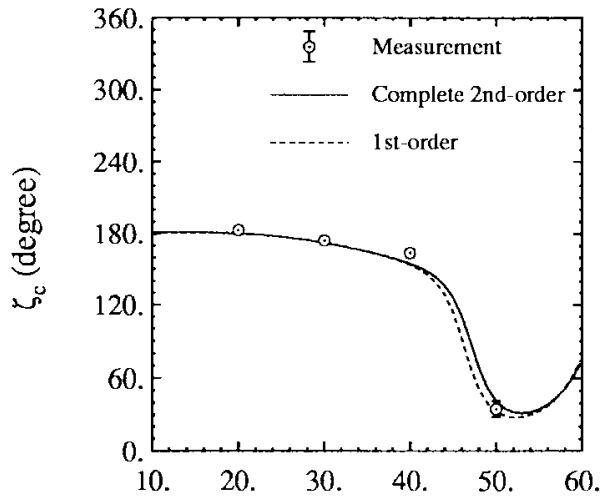

Incidence Angle (degree)

(b)

Fig. 16. Comparison of the measured and calculated copolarized coherent phase difference for a sand layer of thickness (a) $2.52 \mathrm{~cm}$ and (b) $3.53 \mathrm{~cm}$ above a perfectly conducting ground plane at $9.25 \mathrm{GHz}$.

A uniform sand with maximum particle dimension of 0.15 $\mathrm{mm}$ was chosen to minimize the effect of volume scattering from the sand layer. The effective dielectric constant of the sand medium was measured to be $\epsilon_{r}=2.7+i 0.05$. The radar was calibrated polarimetrically using STCT [17]. To generate a desired roughness over the sand surface repeatedly, a template was made. The imprint of the template on the surface generated a rough surface with almost an exponential autocorrelation function with $k s=0.122$ and $k l=2.69$. The surface roughness statistics were measured using a laser ranging system with a range resolution of $0.1 \mathrm{~mm}$. The backscatter measurements conducted for two layers having thicknesses $d=2.52 \mathrm{~cm}$ and $d=3.53 \mathrm{~cm}$ over the angular range $20-50^{\circ}$.

Fig. 15(a) and (b) shows the measured and simulated $\sigma^{0}$ versus incidence angle. All the measured results are shown to be in a very good agreement with the complete second-order solution except for the cross-polarized responses at $\theta=50^{\circ}$. For these cases we were limited by the system noise floor. Fig. 16(a) and (b) shows the response of the copolarized coherent phase difference as a function of incidence angle. Both the first-order and second-order solutions are shown and 


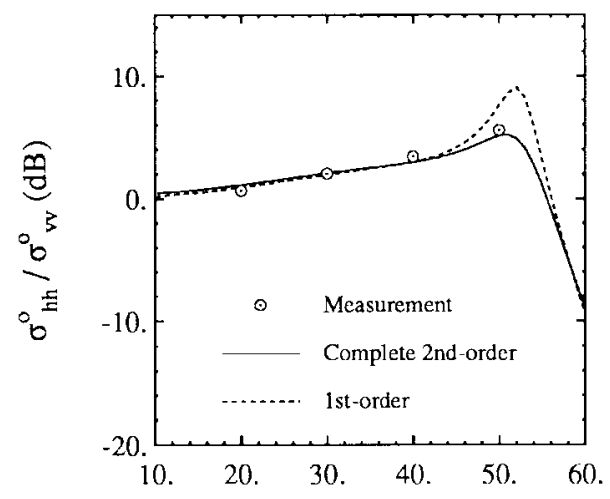

Incidence Angle (degree)

(a)

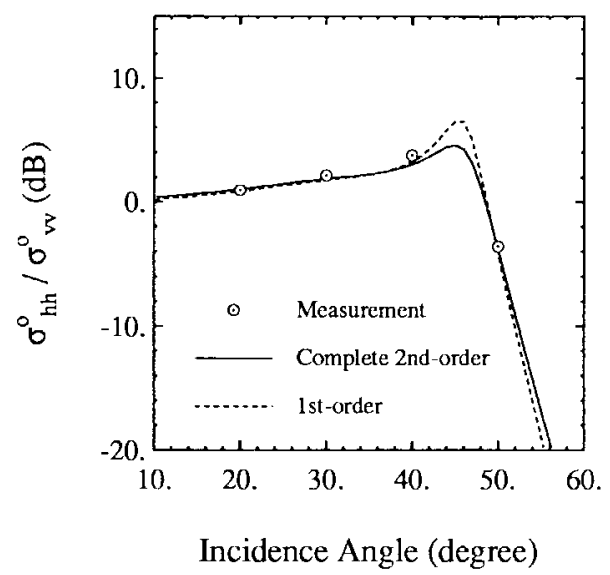

(b)

Fig. 17. Comparison of the measured and the calculated $\sigma_{h h h h}^{o} / \sigma_{v v v v}^{o}$ for a sand layer of thickness (a) $2.52 \mathrm{~cm}$ and (b) $3.53 \mathrm{~cm}$ above a perfectly conducting ground plane at $9.25 \mathrm{GHz}$.

it is obvious that the second-order contribution is insignificant at angles below $40^{\circ}$.

Fig. 17(a) and (b) compare the measured and theoretical ratio of $\sigma_{h h h h}^{0} / \sigma_{v v v v}^{0}$ versus incidence angle. Here it is shown that at high-incidence angles first-order results are incapable of accurate prediction of backscattering coefficients whereas the second-order solution provide satisfactory results. Fig. 18(a) and (b) shows the measured and calculated copolarized degree of correlation versus incidence angle where a relatively good agreement has been obtained considering the difficulties in the accurate measurement of $\alpha$ [18].

\section{CONClusions}

In this paper, a bistatic polarimetric scattering model for random dielectric surfaces with inhomogeneous permittivity profiles and small surface roughnesses is developed using a perturbation expansion of volumetric polarization current. A complete second-order solution for the backscattering coefficients and the statistics of the phase difference between the elements of scattering matrix is obtained. The validity of the model is verified in a limiting case, where it is shown that the formulation for surface with inhomogeneous permittivity profile reduces to the known formulation for

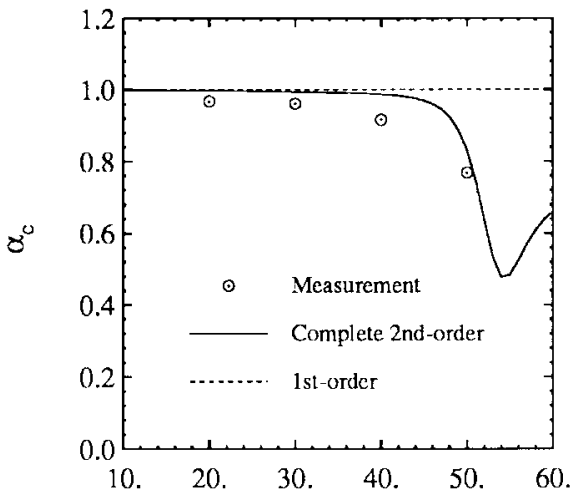

Incidence Angle (degree)

(a)

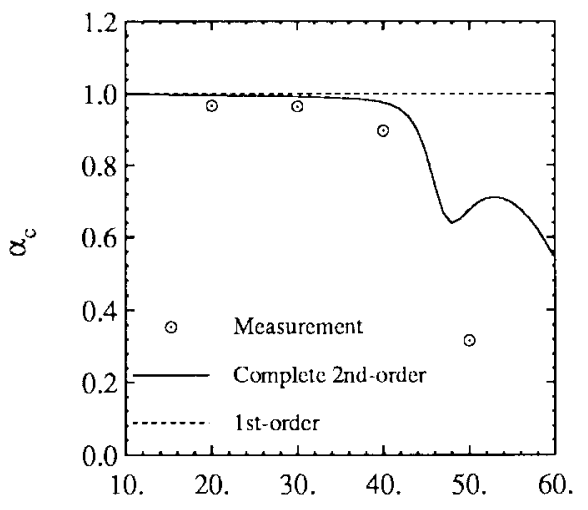

Incidence Angle (degree)

(b)

Fig. 18. Comparison of the measured and the calculated copolarized degree of correlation for a sand layer of thickness (a) $2.52 \mathrm{~cm}$ and (b) $3.53 \mathrm{~cm}$ above a perfectly conducting ground plane at $9.25 \mathrm{GHz}$.

homogeneous rough surfaces. Also, polarimetric backscatter measurements from rough surfaces with known dielectric profiles and roughness statistics were collected and compared with the theoretical calculations. Comparisons with the measured data show excellent agreement. The sensitivity analysis in terms of the surface physical parameters is also performed. It is shown that, in general, the backscatter parameters such as backscattering coefficients and phase-difference statistics, are more sensitive to $k s$ than $k l$. The contribution of the secondorder solution for calculation of $\sigma_{h h h}^{o}$ is more significant than that for the calculation of $\sigma_{v v v v}^{O}$. The contribution of the second-order solution to overall $\sigma_{h h h h}^{\circ}$ can be as high as $2 \mathrm{~dB}$ for $k s \leq 0.3$. It is shown that for continuous inhomogeneous profiles, the backscattering coefficients are insensitive to the variations of moisture content as a function of depth. In other words, the backscattering coefficients of a surface with a continuous soil moisture profile are equal to those of a homogeneous surface having a moisture content equivalent to that of the inhomogeneous profile at the interface. The only backscatter parameter sensitive to moisture profile is the copolarized coherent phase difference $\left(\zeta_{c}\right)$. However, both the backscattering coefficients and phase-difference statistics are very sensitive to step discontinuities in moisture profile. 


\section{REFERENCES}

[1] Y. Oh, K. Sarabandi, and F. T. Ulaby, "An empirical model and an inversion technique for radar scattering from bare soil surfaces," IEEE Trans. Geosci. Remote Sensing, vol. 30, pp. 370-382, Mar. 1992.

[2] S. O. Rice, "Reflection of electromagnetic wave by slightly rough surfaces," Commun. Pure Appl. Mathem., vol. 4, pp. 351-378, 1951.

[3] M. Nieto-Vesperinas, "Depolarization of electromagnetic waves scattered from slightly rough random surfaces: A study by means of the extinction theorem," J. Opt. Soc., vol. 72, no. 5, pp. 539-547, 1982.

[4] G. S. Agarwal, "Interaction of electromagnetic waves at rough dielectric surfaces," Phys. Rev. B, vol. 15, pp. 2371-2383, 1977.

[5] L. Tsang, J. Kong, and R. T. Shin, Theory of Microwave Remote Sensing. New York: Wiley, 1985.

[6] P. Beckmann and A. Spizzichino, The Scattering of Electromagnetic Waves from Rough Surfaces. New York: Pergamon, 1963.

[7] D. Winebrenner and A. Ishimaru, "Investigation of a surface field phase perturbation technique for scattering from rough surfaces," Radio Sci., vol. 20, no. 2, pp. 161-170, Mar./Apr. 1985.

[8] E. Rodriguez and Y. Kim, "A unified perturbation expansion for surface scattering," Radio Sci., vol. 27, no. 1, pp. 79-93, Jan./Feb. 1992.

[9] A. K. Fung and G. W. Pan, "A scattering models for perfectly conducting random surfaces: Model development," Int. J. Remote Sensing vol. 8, pp. 1579-1593, 1987.

[10] A. K. Fung, Z. Li, and K. S. Chen, "Backscattering from a randomly rough dielectric surface," IEEE Trans. Geosci. Remote Sensing, vol. 30, pp. 356-369, Mar. 1992.

[11] T. Chiu and K. Sarabandi, "Scattering solutions for slightly rough surfaces with inhomogeneous dielectric profiles," Radiation Lab. Rep. RL-946, University of Michigan, Ann ARbor, MI, 1997.

[12] K. Sarabandi, "Derivation of phase statistics from the Mueller matrix," Radio Sci., vol. 27, no. 5, pp. 553-560, Sept./Oct. 1992.

[13] M. T. Hallikainen, F. T. Ulaby, M. C. Dobson, M. A. El-Rayes, and L. Wu, "Microwave dielectric behavior of wet soil-Part I: Empirical models and experimental observations," IEEE Trans. Geosci. Remote Sensing, vol. GRS-23, pp. 25-34, Jan. 1985.
[14] E. G. Njoku and J. Kong, "Theory for passive microwave remote sensing of near-surface soil moisture," J. Geophys. Res., vol. 28, pp. 1022-1033, 1990.

[15] F. G. Tricomi, Integral Equations. New York: Intersci., 1957.

[16] R. DeRoo, R. Hartikka, N. Peplinski, and A. Zambetti, Bistatic Measurement Facility User's Manual, University of Michigan, Radiation Lab. Ann Arbor, MI, Aug. 1994.

[17] K. Sarabandi and F. T. Ulaby, "A convenient technique for polarimetric calibration of radar systems," IEEE Trans. Geosci. Remote Sensing, vol. GRS-23, pp. 25-34, Jan. 1985.

[18] K. Sarabandi, Y. Oh, and F. T. Ulaby, "Measurement and calibration of differential Mueller matrix of distributed targets," IEEE Trans. Antennas Propagat., vol. 40, pp. 1524-1532, Dec. 1992.

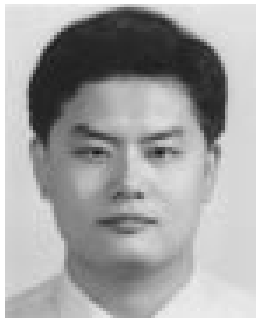

Tsenchieh Chiu was born in Kaohsiung, Taiwan, on November 3, 1968. He received the B.S. and M.S. degrees in electrical engineering from National Taiwan University, Taipei, Taiwan, in 1990 and 1992 , respectively. He is currently working toward the Ph.D. degree in electrical engineering at the University of Michigan, Ann Arbor.

$\mathrm{He}$ is currently a Research Assistant with the Radiation Laboratory, University of Michigan, Ann Arbor. His research interests include microwave remote sensing and calibration of the radar system. 